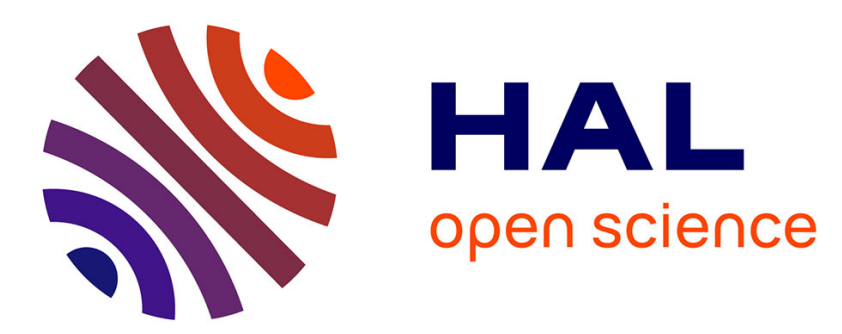

\title{
A Heuristic Approach Applied to Time Reversal MUSIC Method for Soft Fault Location in Noisy Transmission Line Networks
}

\author{
Moussa Kafal, Andréa Cozza
}

\section{- To cite this version:}

Moussa Kafal, Andréa Cozza. A Heuristic Approach Applied to Time Reversal MUSIC Method for Soft Fault Location in Noisy Transmission Line Networks. PhotonIcs \& Electromagnetics Research Symposium (PIERS 2019), IEEE, Jun 2019, Rome, Italy. 9017914, pp 3077-3085, 10.1109/PIERSSpring46901.2019.9017914 . hal-02119869

\section{HAL Id: hal-02119869}

https://hal-centralesupelec.archives-ouvertes.fr/hal-02119869

Submitted on 11 Dec 2020

HAL is a multi-disciplinary open access archive for the deposit and dissemination of scientific research documents, whether they are published or not. The documents may come from teaching and research institutions in France or abroad, or from public or private research centers.
L'archive ouverte pluridisciplinaire HAL, est destinée au dépôt et à la diffusion de documents scientifiques de niveau recherche, publiés ou non, émanant des établissements d'enseignement et de recherche français ou étrangers, des laboratoires publics ou privés. 


\title{
A Heuristic Approach Applied to Time Reversal MUSIC Method for Soft Fault Location in Noisy Transmission Line Networks
}

\author{
M. Kafal ${ }^{1}$, and A. $\mathrm{Cozza}^{2}$ \\ ${ }^{1}$ CEA, LIST, F-91191 Gif-sur-Yvette Cedex, France \\ ${ }^{2}$ GeePs, CentraleSupelec, Universite Paris-Saclay, 3 \& 11 rue Joliot-Curie, 91192 Gif-sur-Yvette, France
}

\begin{abstract}
Time-Reversal multiple signal classification (TR-MUSIC) has emerged as a promising technique to locate multiple soft faults in complex wire networks, thanks to its location accuracy and sub-millimeter resolution. The surprising ability to operate using continuous wave excitations even at low frequencies makes its readily adaptable to live wire testing. However, real-life networks are always surrounded by different sources of noise, an issue which is rarely addressed in the domain of fault detection and location. Intrinsically, TR-MUSIC capable of resolving multiple scatterers in open media has shown resolution degrading problems in the presence of perturbations. Accordingly, we will study in this paper the effect noise can bring on the fault location accuracy in different complex wire networks. This will be followed by proposing a heuristic clustering approach which will enable returning good estimates of the fault location at signal to noise ratio (SNR) well below $5 \mathrm{~dB}$.
\end{abstract}

\section{INTRODUCTION}

Nowadays, equipments designed to manage system (transportation, machinery, etc.) maintenance are considered analogous to health care systems intended to manage human health. Although some elements are designed to be highly reliable and fault-free long-lasting, fatal consequences are the outcome in case of failure. Therefore, such elements require special reliability considerations [1]. Transmission line cables in different application networks provide a particularly salient example.

Unlike most elements composing electrical equipments which are usually installed in clean and sealed environments, electrical wires are generally deployed in inaccessible and harsh conditions $[2,3]$. Accordingly, they are readily exposed to suffer from anomalies leading to the appearance of either hard faults (open or short circuits) that are generally the origin of incidents or soft faults (e.g., chafing, insulation damage, etc.), which are usually minor alterations that affect a cable without impeding its nominal functions. Notably, the latter kind of faults have earned attention as their evolution could be continuously monitored, in order to ideally intervene before their development into hard faults, thus avoiding a sudden system collapse .

Although, several variants of reflectometry methods using several diagnosis systems in parallel have been recently introduced and developed, the weak reflectivity of soft faults makes this class of diagnostic techniques poorly effective $[2,3,4,5,6,7,8,9]$. This state of affair worsens whenever soft faults occur within multiple-branch networks. In fact, multiple reflections caused by junctions and cable ends can easily mask the soft fault's signature, which is expected to aggravate in the presence of noise. To cope with these constraints, recent studies revolving around time reversal (TR) techniques have been applied with success to the detection and location of soft faults in networks under test (NUT) of a varying degree of complexity [10, 11, 12].

In particular, TR-multiple signal classification (TR-MUSIC) has recently been shown to enable super-resolved estimates of a fault location while using continuous-wave excitations, powering sub-wavelength imaging techniques [13]. Resolutions of the order of the millimeter have been demonstrated testing NUTs with signals below $10 \mathrm{MHz}$. Similar spatial resolutions would require test signals covering bandwidths spanning several $\mathrm{GHz}$ when using reflectometry techniques.

In fact, studies have shown that unlimited resolution can be achieved by TR-MUSIC in noiseless environments. But real-life NUTs are always affected by some kind of background noise that can greatly degrade the quality of sub-wavelength imaging. In their investigations, [16] and [17] have proven that for any kind of noise in open media, the resolution of the MUSIC estimator can not be systematically achieved and a greater deterioration is obtained on low frequencies. Therefore, the present paper aims at exploring the influence of noise level on both the imaging resolution and the accuracy of faults position. 


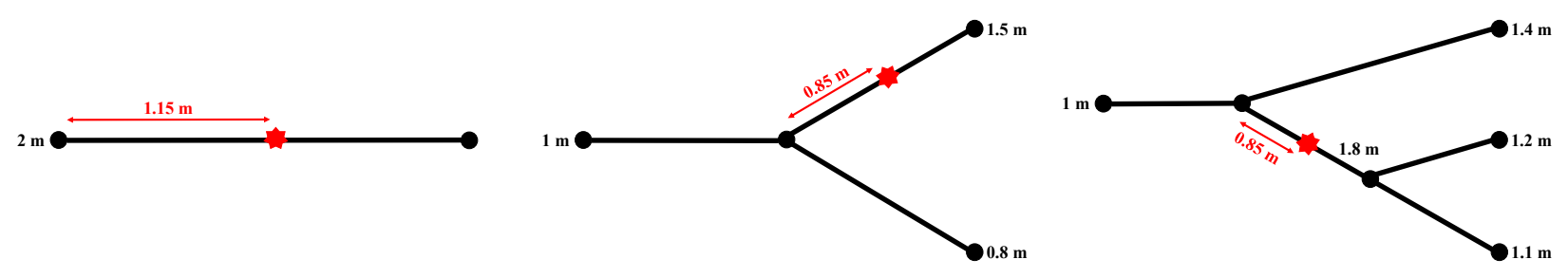

Figure 1: Topologies of the networks used for the TR-MUSIC experimental validation affected by a single soft fault: single branch; single junction and double junction NUTs (from left to right).

The structure of this paper is as follows. Section 2 reviews the basic aspects of the TR-MUSIC method applied to guided wave propagation along wiring networks for the purpose of soft-fault detection. Sec. 3 is dedicated to present a performance analysis of noise on the resolution feature of TR-MUSIC on several network topologies. In this respect, the study will be accomplished on a wide range of frequency samples in an effort to analyze the noise impact on low and high frequencies. The resolution deterioration obtained at low SNR will be resolved by proposing a heuristic histogrambased approach in section 4 which will be assessed on different complexity networks on a range of low to high SNRs. Section. 5 will investigate the results and provide a performance analysis. Finally, sec. 6 concludes this paper with final remarks and future perspectives.

\section{TR-MUSIC FOR SOFT FAULT DIAGNOSIS IN TRANSMISSION LINE NETWORKS}

The basic operations involved in the use of TR-MUSIC technique in fault location, are here recalled; the interested reader should refer to $[12,13]$ for more details. All subsequent quantities are function of the frequency, except where otherwise indicated, hence the frequency variable will be neglected.

The proper application of TR-MUSIC relies on the availability of the NUT's scattering matrix of an $N$ testing port network which can either be computed numerically or measured experimentally using a vector network analyzer (VNA). On the other hand, dealing with soft faults necessitates a baselining approach, i.e., taking the difference between the response (scattering matrices) of the NUT, containing an eventual fault $\mathbf{S}_{\mathbf{f}}$, and a reference response of a healthy version of the network $\mathbf{S}_{\mathbf{h}}[18,19]$. In fact, applying the difference system $\mathbf{S}=\mathbf{S}_{\mathbf{f}}-\mathbf{S}_{\mathbf{h}}$ is multifaceted as it is conceptually fundamental to allow considering the fault as a secondary source, an important feature for the employment of TR techniques to fault detection problems. Besides, it not only permits removing the pseudo echoes generated by impedance discontinuities like junctions but also increases the maximum number of faults $M$ that TR-MUSIC can detect, which is theoretically limited to $N-1$.

The TR operator (TRO) is the central key of most TR variants which is obtained from the scattering matrix as $\mathbf{K}=\mathbf{S}^{\dagger} \mathbf{S}$, where the superscript $\dagger$ is the Hermitian transpose. TR-MUSIC is designed to locate $M$ faults from the knowledge of $\mathbf{K}$ under the condition that $N>M$. In particular, the eigenvalue decomposition (EVD) of the TRO forms the basis of the TR-MUSIC method by employing the null subspace $\mathcal{N}$ of $b f K . \mathcal{N}$ is formed from eigenvectors deemed to have negligible eigenvalues $\mathcal{N}=\operatorname{span}\left\{\mathbf{u}_{\mathbf{i}}: \lambda_{\mathbf{i}}<\lambda_{\mathbf{t h}}\right\}$, with $\lambda_{i}$ and $\mathbf{u}_{\mathbf{i}}$ being the eigenvalues and their corresponding eigenvectors, respectively; $\lambda_{t h}$ is set by analyzing the scree plot of the eigenvalues of K. Accordingly, each fault position can be spotted by observing the singularities obtained in the so-called pseudo-spectrum $\Phi\left(X_{f}\right)$ at each position $X_{f}$ given by:

$$
\Phi\left(X_{f}\right)=\frac{1}{\sum_{i}\left|\mathbf{u}_{\mathbf{i}}^{\dagger} \mathbf{g}\left(\mathbf{X}_{\mathbf{f}}\right)\right|^{2}},
$$

with $\mathbf{g}\left(\mathbf{X}_{\mathbf{f}}\right)$ being the Green function phase patterns, computed at any position $x_{f}$ of the medium. Noteworthy, a Green function is a complex mathematical tool which represents a wave varying both in time and space. In other words, it represents a propagating wave from a certain point to another one where only the phase pattern of the wave is taken into account. In our case study, the Green function is a voltage computed at each position $x_{f}$ of the line by numerically injecting test signals to a healthy version of the NUT. 


\section{TR-MUSIC PERFORMANCE IN THE PRESENCE OF NOISE}

In [12] and [14], the authors proved that TR-MUSIC ensured a sub-millimeter resolution while using relatively low test frequencies. Although spurious ghosts attributed to the periodicity of guided wave propagation appeared in the pseudospectrum, imposing a maximum test frequency $F_{\max } \lesssim v /(2 L)$ solved the issue, with $L$ being the maximal length found through an NUT, and $v$ being the speed of the wave. Therefore, it is very likely that the most striking feature of TR-MUSIC is demonstrated by its ability to locate multiple soft faults using continuous (single frequency) waves even in complex branched networks; an advantage outperforming all rivals in this domain.

Despite the fact that a vast majortity of soft fault location techniques limit thier validation to controlled noiseless environments, pratical implementation requires a performance analysis against different kinds of perturbations. For this reason, it would be interesting to quantify and assess the response of TR-MUSIC technique in the presence of noise.

\subsection{Addition of AWGN}

TR-MUSIC operates by comparing the phase patterns of the noise space $\mathcal{N}$ eigenvectors derived from the NUTs scattering matrix with the phase of the Green function vector at each position of the tested network. In fact, the TRO computed from the scattering matrices of an NUT is usually noiseless since measurements are collected at high signal-to-noise ratio $(\mathrm{SNR} \approx 70 \mathrm{~dB})$ at the level of the VNA. Thus, noise will be added numerically to the measured data. As such, it is convenient to compute the SNR at the level of the measured scattering matrices, where noise will be applied to all elements of the baselined matrix $\mathbf{S}$ before proceeding to the post-processing stage. We will consider an analysis based on the presence of an additive white Gaussian noise (AWGN), which does not bring any limitation to our conclusions, as the same analysis could be carried out considering other types of noise. The choice of an AWGN is just motivated by the simplicity of the related analysis.

In order to better understand the process of noise addition, we shall first note that each scattering matrix $\mathbf{S}_{i}(i=f$ or $h)$ is independently collected at each frequency sample of the total considered bandwidth. Consequently, different levels of noise will be added to $\mathbf{S}$ at each frequency sample so that a fixed SNR value could be defined on the total bandwidth. In other words, if $\mathbf{S}^{f_{k}}$ is the scattering matrix at a frequency sample $f_{k}$, and $P_{s i g}^{f_{k}}$ is the average power computed from all components of the corresponding scattering matrix as given by eq. 2 , then for a value $x d B$ of the SNR, the noise power $P_{\text {noise }}^{f_{k}}$ can be calculated according to eq. 3. As a result, the average power $P_{\text {sig }}^{f_{i}}$ becomes the square of the Frobenius norm of $\mathbf{S}^{f_{k}}$ as given in eq. 2, with $n$ being the dimension of $\mathbf{S}^{f_{k}}$.

$$
P_{s i g}^{f_{k}}=\frac{1}{2 n} \sum_{i=1}^{n} \sum_{j=1}^{n}\left|\mathbf{S}_{\mathbf{i j}}^{\mathbf{f}_{\mathbf{k}}}\right|^{\mathbf{2}}=\frac{\mathbf{1}}{\mathbf{2 n}}\left(\left\|\mathbf{S}^{\mathbf{f}_{\mathbf{k}}}\right\|_{\mathbf{F}}\right)^{\mathbf{2}}
$$

It is noteworthy that the rational behind computing the average value of $\mathbf{S}^{f_{k}}$ as the signal power rather than choosing the maximum value is to avoid the total corruption of weak elements in $\mathbf{S}^{f_{k}}$. In effect, a unique SNR will be defined for $\mathbf{S}$ at all frequency samples of the considered measuring bandwidth. The TR-MUSIC algorithm will then postprocess the measured scattering matrices after having numerically applied AWGN on a range of SNR values.

$$
\mathrm{SNR}_{d B}=\log _{10}\left(\frac{P_{\text {sig }}^{f_{k}}}{P_{\text {noise }}^{f_{k}}}\right)
$$

\subsection{Effect of Noise on the Pseudo-spectrum}

In our study, we will examine the same NUTs considered in the experimental validations of the TRMUSIC method in [12] which were implemented using standard $50 \Omega$ coaxial cables. Three different complexity networks will be accounted starting from the simple single-branch configuration passing by the single-junction structure and finally to the complex double-junction network topology as depicted in Fig. 1. A single soft fault will be inserted in all NUTs which is created by applying a crushing force along an area of $2 \mathrm{~mm}$ to a $30 \mathrm{~cm}$ semi-rigid coaxial cable. The scattering matrices for each tested network were measured using a Rohde \& Schwarz ZVB8 VNA on a bandwidth ranging from $1 \mathrm{MHz}$ to $500 \mathrm{MHz}$ and a sampling frequency step of $1 \mathrm{MHz}$. Although, this might 

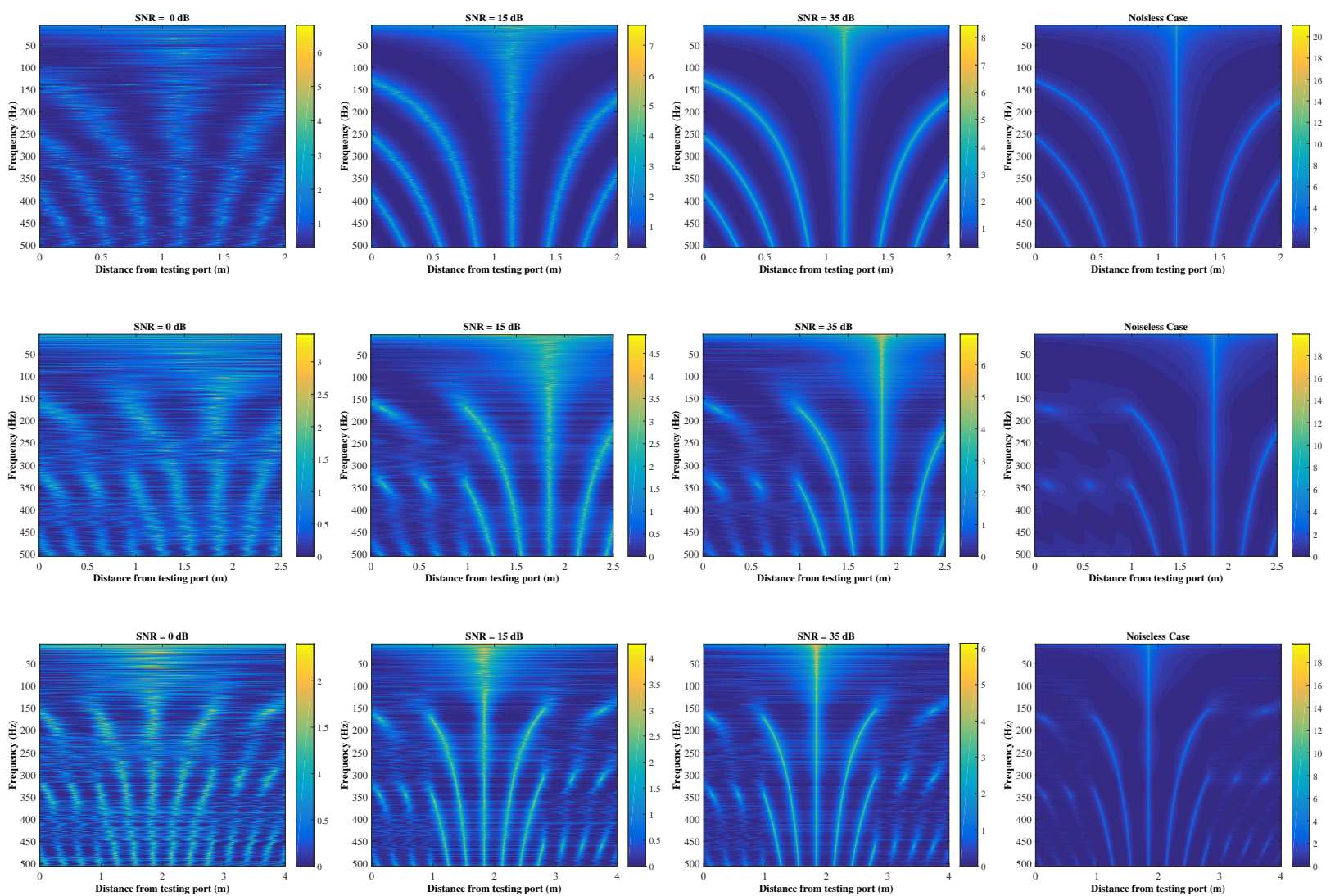

(a)

(b)

Figure 2: Pseudo-spectra of the single branch, single junction and double junction NUTs containing a single soft fault, after applying the TR-MUSIC method on the experimental data collected on a frequency range from $1 \mathrm{MHz}$ to $500 \mathrm{MHz}$ for the noiseless case and after applying AWGN on different values of the SNR listed on the top of each figure.

seem awkward as TR-MUSIC has shown promising results based on single frequency measurements, but it would be useful to study the effect noise can bring on low and high frequencies.

In our investigation, AWGN was numerically added to all the NUTs' measured baselined scattering matrices while considering SNR values ranging from the worst $0 \mathrm{~dB}$ case till $35 \mathrm{~dB}$ with a step of $5 \mathrm{~dB}$. Thereafter, TR-MUSIC method is applied in the post-processing to the resulting noisy scattering matrices of the three studied networks. Figs. 2 show the pseudospectra obtained at different values of the SNR along with the noiseless case for the sake of comparison which is obtained by setting the VNA input power at $0 \mathrm{dBm}$ which ensures a strong rejection of noise.

It would be interesting to briefly recall the process of analyzing a pseudospectrum resulting from the application of TR-MUSIC on an NUT. So, if we consider any of the noiseless pseudospectra of Figs. 2, we can realize a number of singularities appearing at each frequency with only one keeping constant at the fault position which is marked by a vertical line. The remaining singularities are periodically spaced ghosts by half a wavelength caused by the periodicity of wave propagation of harmonic signals. Nevertheless, complexifying the network contributes in breaking down this periodicity due to the presence of junctions and branches as depicted in Figs. 2(b),\&(c) of the single and double junction networks.

Now if we reconsider again the pseudospectra for different levels of noise, several points need to be commented. First of all, despite the high resolution merit of TR-MUSIC, the presence of strong noise at low SNRs has greatly devastated this feature. Observing the pseudospectra at SNRs well below $15 \mathrm{~dB}$ shows a dramatic degradation of the sub wavelength resolution. Regrettably, resolving the fault's singularity becomes a hard task whether at low frequencies which are less tolerant to noise or at high frequencies where distinguishing the fault's singularity from ghosts becomes impossible. Besides, it can be noted from the pseudospectra that singularities whether corresponding to the fault or ghosts have been significantly biased and symmetrically scattered around the true position of their corresponding singularities. Accordingly, in the case of noisy measuring environments, 
applying the TR-MUSIC on larger bandwidths comprising low and high frequencies with more frequency samples would not only compensate the deterioration obtained on low frequencies and the ambiguity overwhelming high frequencies but could also average the noise leading to better inviolability to noise effects.

On the other hand, it is also important to realize that the constellation of points corresponding to the fault's position is not anymore pillar shaped, rather it gets distorted and becomes much wider at its top where frequencies are still low. Notwithstanding, we can still visually distinguish the constellation of points corresponding to the fault from those of the ghosts, which have been also affected by this distortion, and accordingly have a rough estimation of the fault's position even at low SNRs. Noteworthy, the distortion seems to have faded at high frequencies.

Therefore, it would be extremely useful to find a suitable algorithm that can automatically distinguish and separate the constellation of points corresponding to the fault's position from existing ghosts. Particularly, this would enable obtaining precise estimates of the fault's position thus overcoming the problem noise that led to the scattering and scrambling of singularities.

\section{FAULT LOCATION HEURISTIC APPROACH}

To be able to propose a suitable approach, interpreting the spreading pattern of the scattered points would promote this objective. Let us consider the case of the single-branch NUT at an SNR value of $10 \mathrm{~dB}$, the histogram of Fig. 3 shows the distribution of a dataset containing all singularity positions of the network. Markedly, the heaviest density (25\% of the total singularity locations) happened to lie within a bin width of $10 \mathrm{~cm}$ centered at the fault position $(1.15 \mathrm{~m}$ from the reference testing port). The remaining points were distributed all over the network positions prior to the presence of ghosts. On the other hand, one can argue that depending on the width of the bin a different precision of the fault's position could be obtained. In fact, this might be true as long as a specific algorithm is not defined, but in our case study a heuristic histogram-based approach will be proposed.
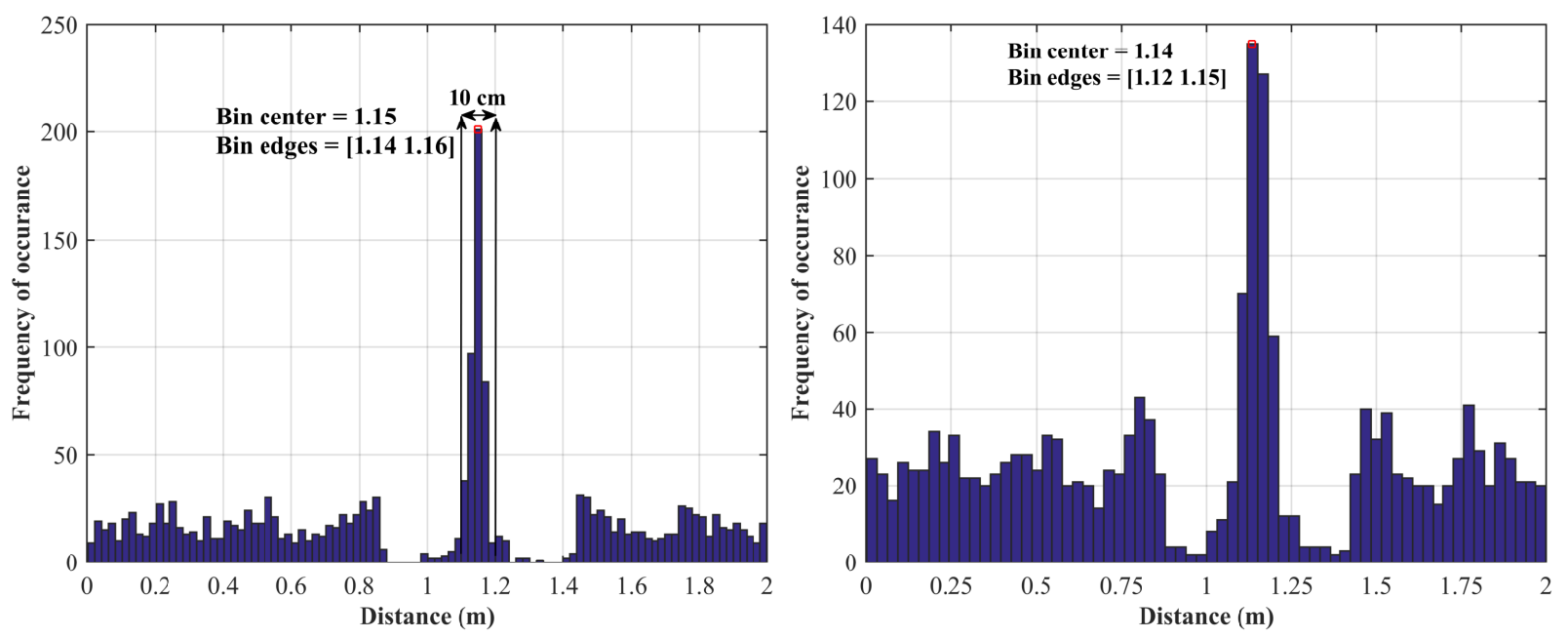

Figure 3: The histogram of the dataset of singularity Figure 4: A histogram plot of the dataset of Fig. 3 positions corresponding to the pseudo-spectrum of after setting a bin width of $0.025 \mathrm{~m}$ prior to the obthe single branch NUT at $10 \mathrm{~dB}$.

tained maximal ghost interval $\mathrm{W}=0.25 \mathrm{~m}$

Based on the fact that the worse ghost scenario occurs in the single branch network configuration [12], and knowing that ghosts appear as a multiple of half the wavelength, we can expect that the maximum number of ghosts including the pillar pointing on the position of the fault is achieved on the maximal frequency step. In other words, for a single branch of length $L$ and operating at a maximum frequency $F_{\max }$, the maximum number of ghosts is

$$
N_{g h}=\frac{L}{\lambda / 2}
$$

with $\lambda=v / F_{\max }$ being the wavelength at $F_{\max }$. Consequently, the maximal distance between a ghost and another is $W=L / N_{g h}$. The previous discussion and results can be translated into a heuristic histogram-based algorithm whose steps are summarized in what follows: 


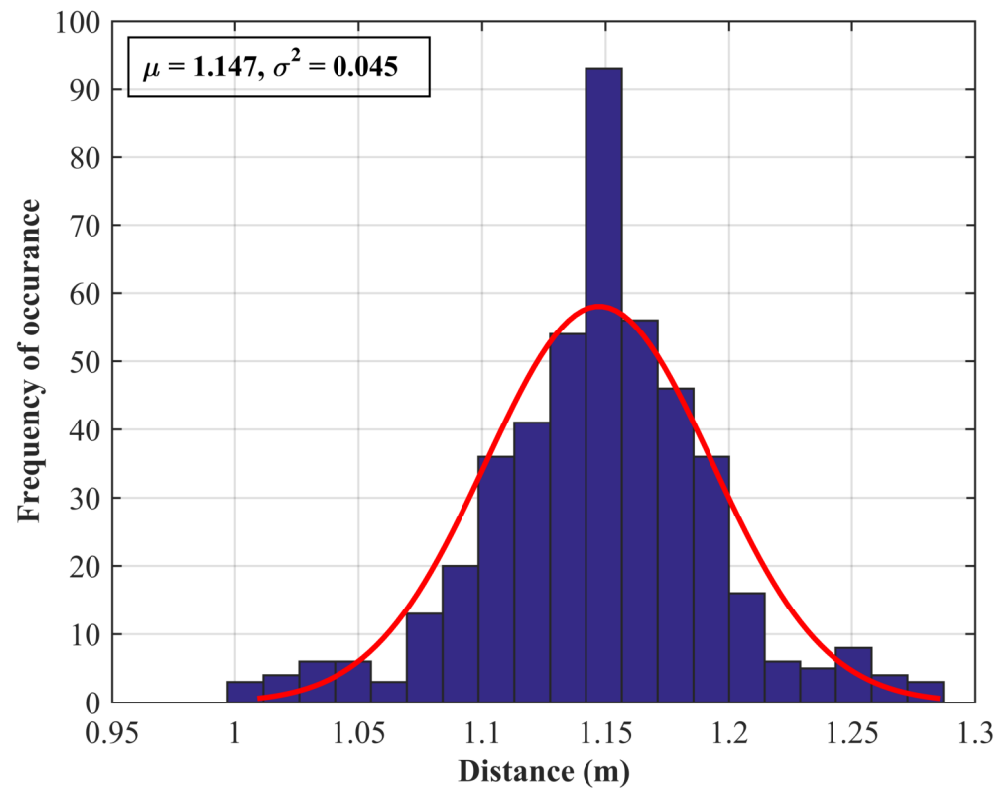

Figure 5: The distribution of singularity positions corresponding to the pseudo-spectrum of the single branch NUT in an interval of $0.25 \mathrm{~m}$ around the preliminary fault's position referring to $1.14 \mathrm{~m}$.

1. Collect all positions of singularities present in the pseudospectrum in a dataset;

2. based on the knowledge of $F_{\max }$ and the total length of the observed path $L$, compute $N_{g h}$ and $W$;

3. plot the data set in a histogram whose bin width $W_{b}=W / M$, with $M$ being a constant;

4. the bin center corresponding to the most frequent bin would be a preliminary estimate $P_{f}^{p r}$ of the fault's position;

5. the estimated fault position $P_{f}^{e s}$ is the mean value of the singularity locations lying in an interval $W$ and centered at $P_{f}^{p r}$.

In order to better understand the proposed fault location algorithm, we will reconsider the $2 \mathrm{~m}$ single-branch NUT at an SNR value of $10 \mathrm{~dB}$. According to eq. 4, the maximum number of ghosts is $N_{g h}=8$ for a maximum frequency of $F_{\max }=500 \mathrm{MHz}$. Accordingly, $\mathrm{W} \approx 0.25 \mathrm{~m}$ and a bin width $W_{b}=W / 10=0.025 \mathrm{~m}$ is set. It is important to note here that the reason behind choosing $W$ as an onset interval is to try as much as possible to collect only singularity positions related to the fault's position otherwise a larger $W$ would probably pollute our data with peak locations from the ghosts. The corresponding histogram is presented in Fig. 4 where the most frequent bin occurs at a bin center of $1.14 \mathrm{~m}$ with a bin width of $\approx 0.25 \mathrm{~m}$. As a result, $P_{f}^{p r}=1.14 \mathrm{~m}$ will form a preliminary estimate of the fault location. This will be followed by collecting all the singularity locations centered at $P_{f}^{p r}$ within an interval $W \approx 0.25 \mathrm{~m}$. The distribution of this new dataset is shown in the histogram of Fig. 5, where we can notice that they have a bell shaped distribution, whose mean value $\mu=1.147 \mathrm{~m}$ reflects the estimated position of the fault.

With this in mind, according to the law of large numbers (LLN) in probability theory [20], the sample average converges almost surely to the expected value at a large number of realizations. Therefore, 1000 different noise realizations are considered at each SNR value where the suggested histogram-based algorithm in sec. 4 will be repeated to return an estimate fault position $P_{f}^{e s}$ at each of these realizations. Indeed, this would return a better, more precise estimation of the fault's position at each specified value of the SNR.

Meanwhile, observing the distribution of $P_{f}^{p r}$ over all the noise realizations for a given value of the SNR would be rather interesting. The cumulative distribution function (CDF) forms a proper tool for this purpose. 


\section{RESULTS AND DISCUSSION}

The CDFs in Fig. 6 (a) are computed for several values of the SNR for the single branch NUT. It can be noted that the steepness of the CDF increases with an increase in the SNR, which is accompanied by a faster convergence towards the eventual position of the fault. In this event, the same result is obtained with the single and double-junction NUTs where the corresponding CDFs are shown in Fig. 6 (b) and (c). With this in mind, Fig. 7 compares the CDFs of the three NUTs computed at an SNR value of $0 \mathrm{~dB}$, where it can be inferred that increasing the network's complexity is accompanied by an increase in the steepness of the curve. Accordingly, a faster convergence towards the actual position of the fault is obtained. In brief, this result is expected as the appearance of ghosts is broken down by the presence of junctions prior to the increase in the complexity of the network. In other words, less singularity locations are obtained in the data of the corresponding pseudo-spectrum starting from the position of the junction.

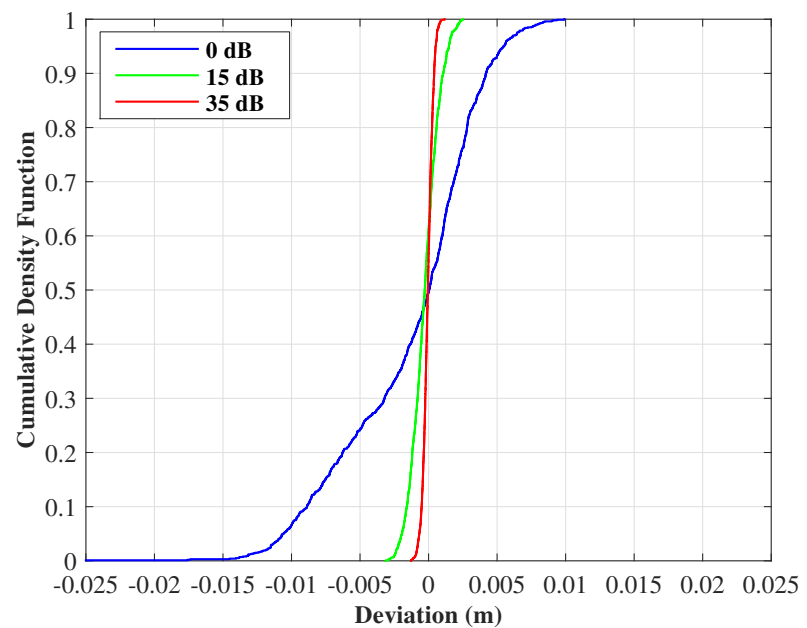

(a)

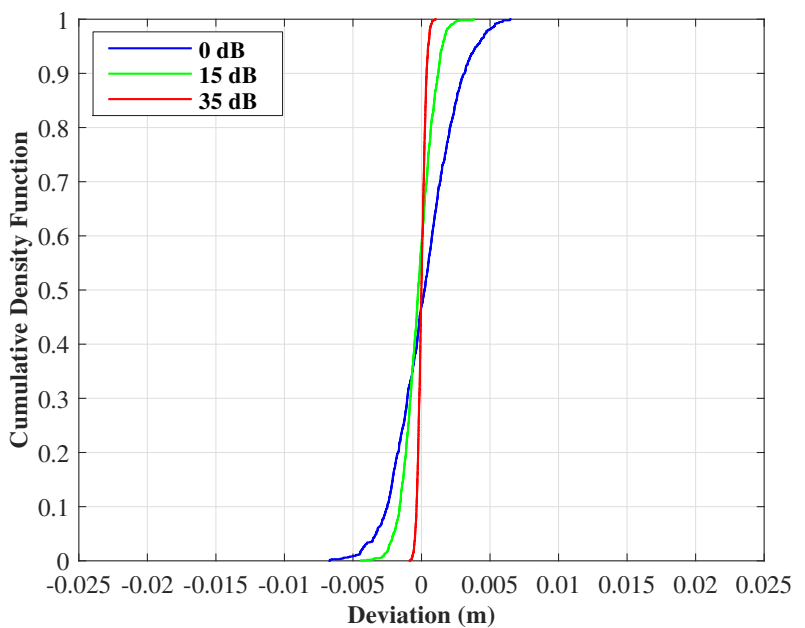

(b)

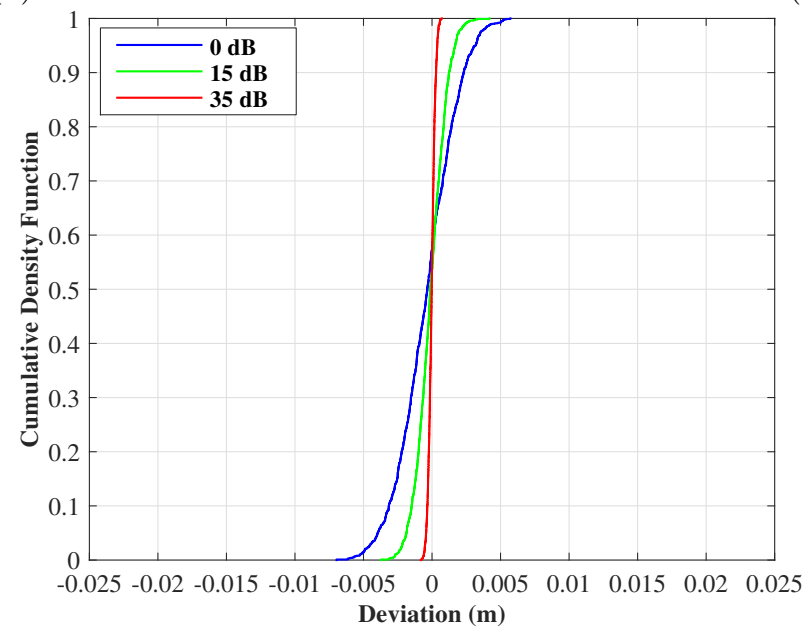

(c)

Figure 6: The cumulative distribution function $(\mathrm{CDF})$ of the deviation of the faults's position for different values of the SNR in the (a): single-branch network configuration, (b): single-junction NUT and (c): doublejunction network.

In order to quantify the variation of the estimated fault's position for a given SNR, its mean value is observed along with its upper and lower bound estimates. A 95\% confidence interval is considered, i.e. the significance level is set to 0.05 where the lower and upper bound estimates are taken at a $2.5 \%$ and $97.5 \%$ of the CDF corresponding to the studied network at a predefined SNR. To better clarify this point, Fig. 8 shows the CDF of the single branch NUT at $0 \mathrm{~dB}$, where the corresponding mean value of the fault's position is $1.149 \mathrm{~m}$, with a lower bound limit of $1.1385 \mathrm{~m}$ and an upper bound limit of $1.1565 \mathrm{~m}$ for a $95 \%$ interval of confidence. Accordingly, the evolution of these values is observed for all tested NUTs as presented in Fig. 9. It can be noticed that for a low 

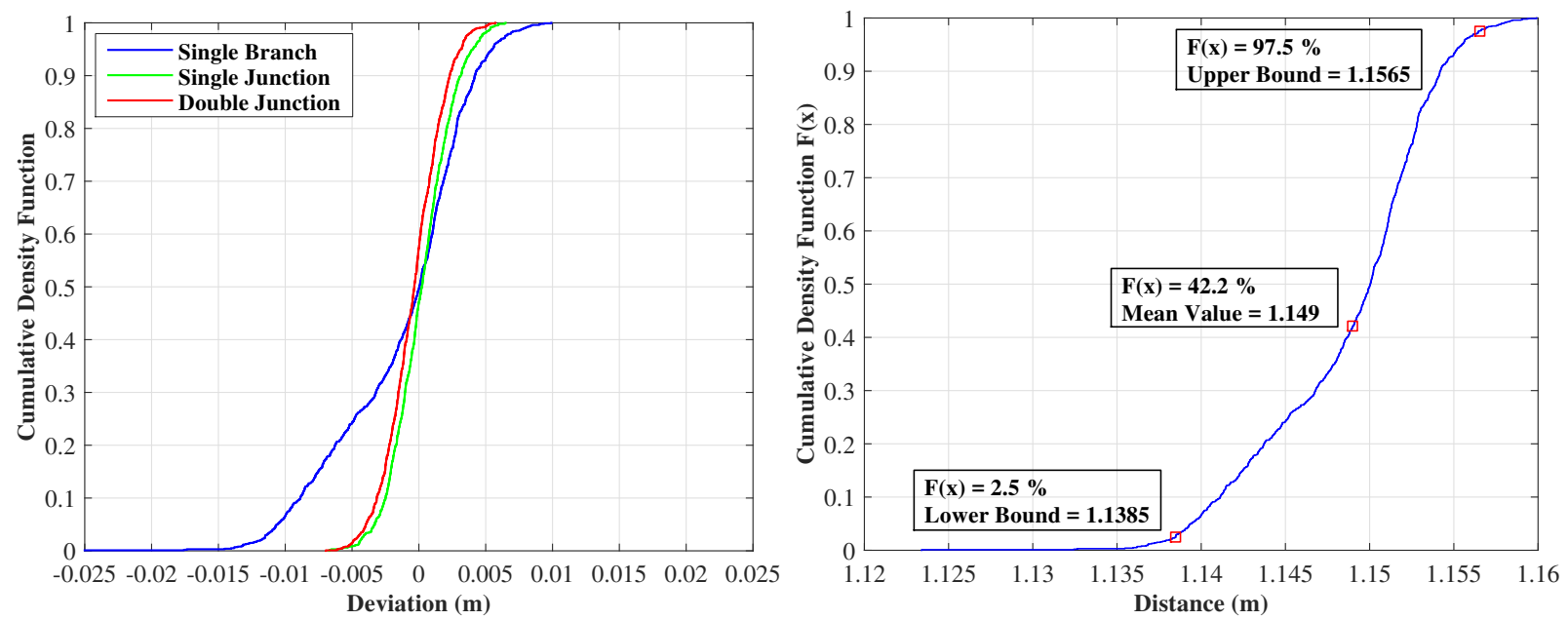

Figure 7: The CDF of the deviation of the faults's Figure 8: The CDF of the deviation of the faults's position for an $\mathrm{SNR}=0 \mathrm{~dB}$ comparing the perfor- position for an $\mathrm{SNR}=0 \mathrm{~dB}$ of the single branch NUT mance with an increased network's complexity.

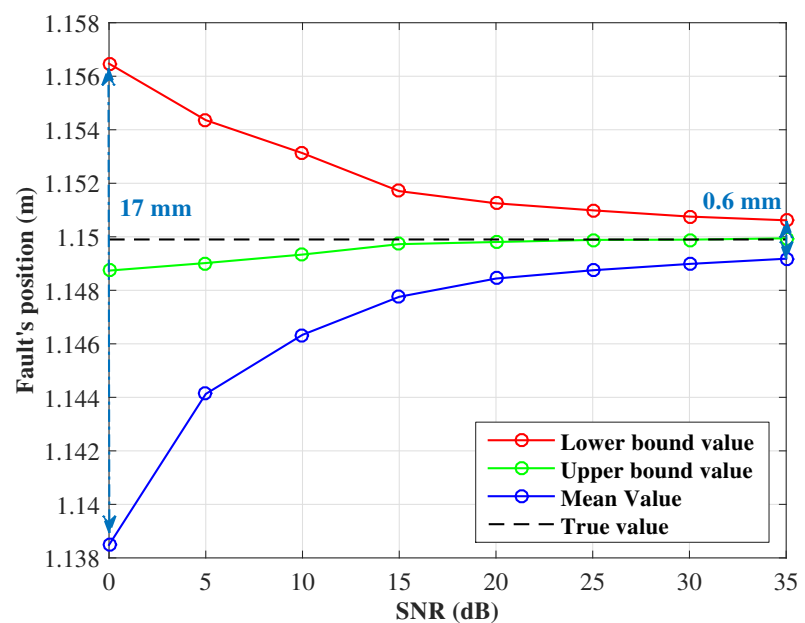

(a)
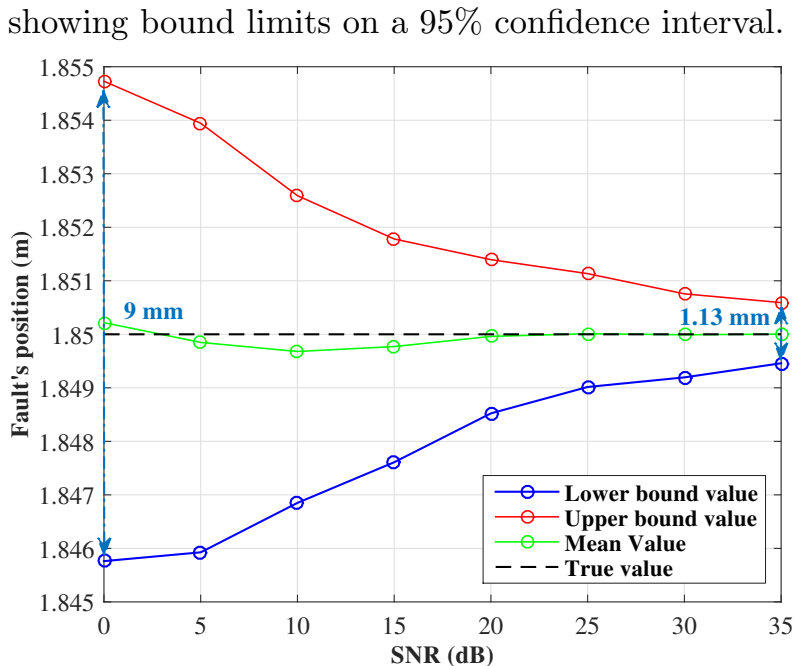

(b)

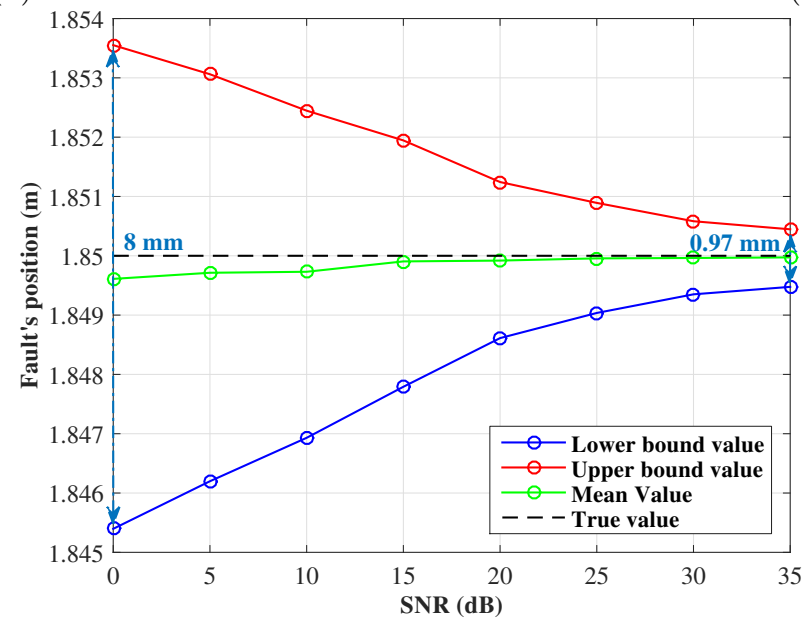

(c)

Figure 9: Performance analysis of the heuristic approach in terms of the estimated fault location error for different values of the SNR in the (a): single-branch network configuration, (b): single-junction NUT and (c): double-junction network.

SNR value of $0 \mathrm{~dB}$, the difference between the upper and lower bounds decreased from $17 \mathrm{~mm}$ for 
the single-branch configuration to $9 \mathrm{~mm}$ for the single-junction NUT and reaching to merely $8 \mathrm{~mm}$ for the double-junction network. Specifically, this improvement in the difference with an increased network complexity may be returned to the accompanied ghost degradation. On the other hand, a significant expected decrease in this difference is observed with an increase in the SNR reachning to no more than $1 \mathrm{~mm}$ at $35 \mathrm{~dB}$. Additionally, the SNR increase is accompanied by a convergence in the fault positions mean value towards the eventual location of the fault.

It shall be worthy to note that, TR-MUSIC with the proposed heuristic approach has provided an effective robustness with noisy data where precise estimates of the fault's position were returned at low SNR. Most importantly, the error didn't exceed the range of $2 \mathrm{~cm}$ at corrupted data of $0 \mathrm{~dB}$, a striking result in the domain of fault detection and location.

\section{CONCLUSION}

This paper has investigated the performance of TR-MUSIC in the presence of noise. In spite of the high resolution feature of TR-MUSIC, the presence of strong noise at low SNR has shown to greatly devastate this accuracy especially at low frequencies. Nevertheless, high frequencies appeared to be less affected. Under those circumstances, using multiple frequencies on wider bandwidths has proven not only to overcome this problem but also contribute in averaging the noise over more samples. Consequently, a histogram-based heuristic approach was proposed as a clustering method to try to isolate singularities lying in the neighborhood of the eventual fault position. Results have shown a great robustness and immunity against noisy data. Surprisingly, few millimeters was the maximum position error obtained at low SNRs as $0 \mathrm{~dB}$ even in complex network structures. Under those circumstaces, implementing TR-MUSIC with the histogram-based approach seems to be a promising technique for testing different real-life networks even in high noisy environments as the aerospace industry for example.

Future work will need to deal with more efficient analytical based clustering methods as the K-means or DBSCAN approaches, etc. for automatic integration with the TR-MUSIC method.

\section{REFERENCES}

1. F. Auzanneau, "Wire troubleshooting and diagnosis: Review and perspectives," Progress In Electromagnetics Research B, vol. 49, pp. 253-279, 2013.

2. C. Furse and R. Haupt, "Down to the wire [aircraft wiring]," Spectrum, IEEE, vol. 38, no. 2, pp. 34-39, 2001.

3. C. Furse, Y. C. Chung, C. Lo, and P. Pendayala, "A critical comparison of reflectometry methods for location of wiring faults," Smart Structures and Systems, vol. 2, no. 1, pp. 25-46, 2006.

4. C. Buccella, M. Feliziani, and G. Manzi, "Detection and localization of defects in shielded cables by time-domain measurements with UWB pulse injection and clean algorithm postprocessing," Electromagnetic Compatibility, IEEE Transactions on, vol. 46, no. 4, pp. 597-605, November 2004.

5. P. Smith, C. Furse, and J. Gunther, "Analysis of spread spectrum time domain reflectometry for wire fault location," IEEE sensors journal, vol. 5, no. 6, pp. 1469-1478, 2005.

6. C. Furse, Y. C. Chung, R. Dangol, M. Nielsen, G. Mabey, and R. Woodward, "Frequencydomain reflectometry for on-board testing of aging aircraft wiring," Electromagnetic Compatibility, IEEE Transactions on, vol. 45, no. 2, pp. 306-315, 2003.

7. S. Schuet, D. Timucin, and K. Wheeler, "A model-based probabilistic inversion framework for characterizing wire fault detection using TDR," Instrumentation and Measurement, IEEE Transactions on, vol. 60, no. 5, pp. 1654-1663, May 2011.

8. A. Cozza, "Never trust a cable bearing echoes: Understanding ambiguities in time-domain reflectometry applied to soft faults in cables," Electromagnetic Compatibility, IEEE Transactions on, vol. 61, no. 2, May 2019.

9. A. Cozza, "Low Frequency Model-Based Identification of Soft Impedance Faults in Cables," Instrumentation and Measurement, IEEE Transactions on, November 2018.

10. L. Abboud, A. Cozza, and L. Pichon, "A noniterative method for locating soft faults in complex wire networks," Vehicular Technology, IEEE Transactions on, vol. 62, no. 3, pp. 1010-1019, 2013.

11. M. Kafal, A. Cozza, and L. Pichon, "Locating multiple soft faults in wire networks using an alternative dort implementation," Instrumentation and Measurement, IEEE Transactions on, vol. 65 , no. 2 , pp. 399-406, 2016. 
12. M. Kafal, A. Cozza, and L. Pichon, "Locating faults with high resolution using single-frequency TR-MUSIC processing," IEEE Transactions on Instrumentation and Measurement, vol. 65, no. 10, pp. 2342-2348, 2016.

13. M. Kafal, J. Benoit, A. Cozza, and L. Pichon, "Soft fault diagnosis in wire networks using time reversal concept and subspace methods," Proc. EEETEM, 2017.

14. M. Kafal, J. Benoit, A. Cozza, and L. Pichon, "Soft fault diagnosis in wire networks using time reversal concept and subspace methods."

15. A. J. Devaney, "Super-resolution processing of multi-static data using time reversal and music," J. Acoust. Soc. Am, 2000.

16. M. Fleming, M. Lowe, F. Simonetti, and P. Cawley, "Super resolution imaging: performance studies," in AIP Conference Proceedings, vol. 820, no. 1. AIP, 2006, pp. 736-743.

17. M. Davy, J.-G. Minonzio, J. de Rosny, C. Prada, and M. Fink, "Influence of noise on subwavelength imaging of two close scatterers using time reversal method: Theory and experiments," Progress In Electromagnetics Research, vol. 98, pp. 333-358, 2009.

18. L.A Griffiths, R. Parakh, C. Furse, B. Baker, "The invisible fray: A critical analysis of the use of reflectometry for fray location," IEEE sensors journal, vol. 6, no. 3, pp. 697-706, June 2006.

19. M. Kafal, and J. Benoit, "Baselining: A critical approach used for soft fault detection in wire networks," EEETEM, 2017.

20. E. W. Weisstein, CRC concise encyclopedia of mathematics. CRC press, 2002. 